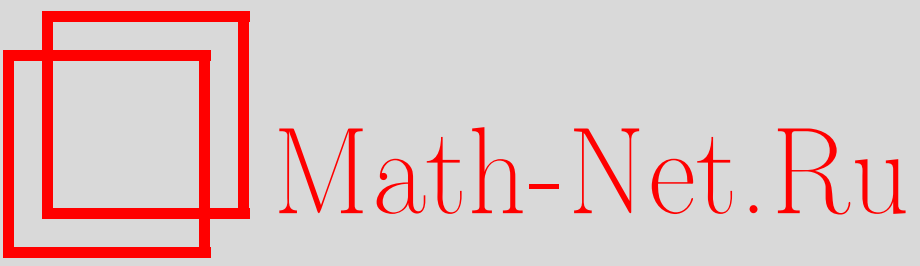

И. Ю. Кадашевич, Ю. И. Кадашевич, А. М. Пейсахов, С. П. Помыткин, Теория пластического течения с перекрестными связями, учитывающая дилатансию и вид напряженного состояния, Вестн. Сам. гос. техн. ун-та. Сер. Физ.-мат. науки, 2002, выпуск 16, 73-74

DOI: https://doi.org/10.14498/vsgtu99

Использование Общероссийского математического портала Math-Net.Ru подразумевает, что вы прочитали и согласны с пользовательским соглашением

http://www.mathnet.ru/rus/agreement

Параметры загрузки:

IP : 54.197 .130 .99

26 апреля 2023 г., 15:51:07 


\section{ТЕОРИЯ ПЛАСТИЧЕСКОГО ТЕЧЕНИЯ С ПЕРЕКРЕСТНЫМИ СВЯЗЯМИ, УЧИТЫВАЮЩАЯ ДИЛАТАНСИЮ И ВИД НАПРЯЖЕННОГО СОСТОЯНИЯ}

Развивается теория пластического течения с перекрестными связями, позволящая описать достаточно тонкие эффекты сложного нагружения, включая проявление эффекта памяти формы. $B$ предлагаемой работе приводятся обобщающие соотношения теории, в которой дополнительно учитываются эффекты дилатансии и влияние вида напряженного состояния. Такой вариант позволяет расширить возможности теории пластичности с перекрестными связями.

Определяющие соотношения для простейшего варианта двухповерхностой теории пластического течения с перекрестными связями имеют вид $[1,2]$

$$
\begin{aligned}
& \frac{d \varepsilon_{i j}^{p(1)}}{d \lambda_{1}}=\frac{T_{i j}^{\prime(1)}}{T_{i}^{(1)}} ; \quad \frac{d \varepsilon_{i j}^{p(2)}}{d \lambda_{2}}=\frac{T_{i j}^{\prime(2)}}{T_{i}^{(2)}} ; \\
& T_{i j}^{\prime(1)}=\sigma_{i j}^{\prime}-C_{11} \varepsilon_{i j}^{p(1)}-C_{12} \varepsilon_{i j}^{p(2)} ; \\
& T_{i j}^{\prime(2)}=\sigma_{i j}^{\prime}-C_{21} \varepsilon_{i j}^{p(1)}-C_{22} \varepsilon_{i j}^{p(2)} ; \\
& T_{i}^{(1)}=\sqrt{T_{i j}^{\prime(1)} T_{i j}^{\prime(1)}} ; \quad T_{i}^{(2)}=\sqrt{T_{i j}^{\prime(2)} T_{i j}^{\prime(2)}} ; \\
& d \lambda_{1}=\sqrt{d \varepsilon_{i j}^{p(1)} d \varepsilon_{i j}^{p(1)}} ; \quad d \lambda_{2}=\sqrt{d \varepsilon_{i j}^{p(2)} d \varepsilon_{i j}^{p(2)}} ; \\
& <\varepsilon_{i j}^{p}>=\frac{\varepsilon_{i j}^{p(1)}+\varepsilon_{i j}^{p(2)}}{2} .
\end{aligned}
$$

Здесь $\sigma_{i j}^{\prime}$ - девиатор тензора напряжений; $\varepsilon_{i j}^{p}$ - девиатор тензора неупругих деформаций; $\varepsilon_{i j}^{p(1)}, \varepsilon_{i j}^{p(2)}$ - девиаторы тензоров локальных неупругих деформаций; <> - знак осреднения; $C_{i j}$ - весовые квазистатистические коэффициенты, характеризующие вклад и взаимодействие локальных поверхностей текучести, причем $\sum_{i, j} C_{i j}=1$.

При учете дилатансии уравнения локальных поверхностей текучести могут быть записаны следующим образом:

$$
\begin{aligned}
& T_{i}^{(1)}+\alpha T_{0}^{(1)}=\sqrt{2} \sigma_{T} ; \\
& T_{i}^{(2)}+\alpha T_{0}^{(2)}=\sqrt{2} \sigma_{T} ; \\
& T_{0}^{(1)}=\sigma_{0}+k_{1} \varepsilon_{0}^{p(1)}+k_{2} \varepsilon_{0}^{p(2)} ; \\
& T_{0}^{(2)}=\sigma_{0}+k_{3} \varepsilon_{0}^{p(1)}+k_{4} \varepsilon_{0}^{p(2)} \\
& d \varepsilon_{0}^{p(1)}=\alpha d \lambda_{1} ; d \varepsilon_{0}^{p(2)}=\alpha d \lambda_{2},
\end{aligned}
$$

где $\sigma_{0}, T_{0}^{(1)}, T_{0}^{(2)} \varepsilon_{0}^{p(1)}, \varepsilon_{0}^{p(2)}$ - первые инварианты тензоров напряжений, локальных активных напряжений и локальных неупругих деформаций, соответственно; $\sigma_{T}$ - предел текучести, а $\alpha$ и $k_{i}$ - параметры материала.

При необходимости в теорию легко можно ввести:

a) различие пределов текучести для каждой поверхности текучести $\left(\sigma_{T}^{(1)} \neq \sigma_{T}^{(2)}\right)$;

б) зависимость пределов текучести от длины дуги траектории неупругого деформирования: $\sigma_{T}^{(1)}=\sigma_{T}^{(1)}\left(\lambda_{1}\right)$ и $\sigma_{T}^{(2)}=\sigma_{T}^{(2)}\left(\lambda_{2}\right)$.

Однако расчеты показывают, что уже простейший вариант теории способен описать основные эффекты, связанные с проявлением эффекта памяти формы.

Группа соотношений теории неупругости с перекрестными связями, учитывающая влияние вида напряженного состояния, основана на расширении теории, предложенной в [3]. Эти уравнения запишем следующим образом:

$$
\frac{d \varepsilon_{i j}^{p(1)}}{d \lambda_{1}}=\frac{L_{i j}^{(1)}}{L_{i}^{(1)}} ; \quad \frac{d \varepsilon_{i j}^{p(2)}}{d \lambda_{2}}=\frac{L_{i j}^{(2)}}{L_{i}^{(2)}} ;
$$




$$
\begin{aligned}
& \frac{L_{i j}^{(1)}}{L_{i}^{(1)}}=\cos \omega_{1} \frac{T_{i j}^{\prime(1)}}{T_{i}^{(1)}}-\sin \omega_{1} \frac{T_{i j}^{*}(1)}{T_{i}^{(1)}} ; \\
& \frac{L_{i j}^{(2)}}{L_{i}^{(2)}}=\cos \omega_{2} \frac{T_{i j}^{\prime(2)}}{T_{i}^{(2)}}-\sin \omega_{2} \frac{T_{i j}^{*(2)}}{T_{i}^{(2)}} ; \\
& \operatorname{tg} \omega_{1}=\frac{\varphi_{1}^{\prime}(\beta)}{\varphi_{1}(\beta)} ; \quad \operatorname{tg} \omega_{2}=\frac{\varphi_{2}^{\prime}(\beta)}{\varphi_{2}(\beta)} ; \quad \omega_{1} \neq \omega_{2} ; \\
& T_{i j}^{*}=T_{i k}^{\prime} T_{k j}^{\prime} ; \quad \sin 3 \beta=-\sqrt{6} \frac{T_{i j}^{\prime} T_{j k}^{\prime} T_{k i}^{\prime}}{T_{i}^{3}},
\end{aligned}
$$

причем функции $\varphi(\beta)$ выбираются в форме $\left(\mu_{1}, \mu_{2}\right.$ - константы материала):

$$
\varphi(\beta)=\left\{\begin{array}{lr}
\exp \left[\mu_{1}\left(\beta-\beta_{*}\right)\right], & \left.-\pi / 6 \leq \beta \leq \beta_{*}\right) \\
\exp \left[\mu_{2}\left(\beta-\beta_{*}\right)\right], & \beta_{*} \leq \beta \leq \pi / 6 .
\end{array}\right.
$$

Авторы данной работы теоретически изучили поведение ряда материалов, обладающих эффектом памяти формы, проявляющих различные механизмы мартенситной неупругости, при сложных температурно-силовых воздействиях, включая пропорциональное, непропорциональное и циклическое нагружения. Особенно интересными для изучения оказались опытные данные о влиянии вида напряженного состояния, опубликованные в [4,5]. В рамках предлагаемой авторами теоретической схемы эти экспериментальные данные удалось описать более просто и естественно, чем по уравнениям теории Лихачева-Малинина [6].

В заключение хотелось бы оттенить некоторые особенности нового варианта теории пластичности с перекрестными связями:

а) в теорию введен механизм работы перекрестных связей, выражающийся в автоматической смене режимов пластического течения в зависимости от истории нагружения и температуры;

б) в теории принято, что при переключении движения с одной активной поверхности на другую или на случай их совместного движения разрешается по определенным правилам [1] скачкообразное изменение параметров теории (резкое изменение постоянных материалов вблизи температур мартенситных превращений типично для всех металлов и сплавов и является одной из причин их аномального механического поведения).

Детальному обсуждению описываемых эффектов будет посвящена специальная публикация.

\section{БИБЛИОГРАФИЧЕСКИЙ СПИСОК}

1. Kadashevich I.Yu., Melsnikov B.E. Phenomenological approach to the superelasticity and shape memory problem description // SPIE. 1998. Vol.3345. P. 176-177.

2. Кадашевич И. Ю., Мельников Б.Е. О возможности использования двухповерхностной теории пластичности при циклическом нагружении // Научно-технические проблемы прогнозирования надежности и долговечности металлоконструкций и методы их решения. СПб., 1998. С. 157-159.

3. Кадашевич И.Ю., Помыткин С.П. Статистическая теория пластичности, учитывающая влияние параметра Лоде // Изв. АН СССР. Механика твердого тела: 1990. N 3. С.91-95.

4. Андронов И.Н., Богданов Н.П., Северова Н.А. Влияние вида напряженного состояния напряженного состояния на характер изотермического деформирования никелида титана // Актуальные проблемы прочности: Науч. тр. XXXIII семинара. Новгород, 1997. С.33-39.

5. Андронов И.Н., Северова Н.А. Влияние вида напряженного состояния на реализацию циклической памяти формы // Заводская лаборатория. 1999. N 6. С.40-47.

6. Лихачев В.А., Малинин В.Г. Структурно-аналитическая теория прочности. СПб.: Наука, 1993. 471 с.

Работа выполнена при поддержке Российского фонда фундаментальных исследований (грант 0101-00229) 